\title{
Bose-Einstein correlations: a study of an invariance group *
}

\author{
A.Bialas and K.Zalewski \\ M.Smoluchowski Institute of Physics \\ Jagellonian University, Cracow \\ and \\ Institute of Nuclear Physics, Cracow
}

February 19, 2018

\begin{abstract}
A group of transformations changing the phases of the elements of the single-particle density matrix, but leaving unchanged the predictions for identical particles concerning the momentum distributions, momentum correlations etc., is identified. Its implications for the determinations of the interaction regions from studies of Bose-Einstein correlations are discussed.
\end{abstract}

PACS numbers 25.75.Gz, 13.65.+i

Bose-Einstein correlations, interaction region determination.

\section{Introduction}

Much work is being done on Bose-Einstein and Fermi-Dirac correlations in multiparticle production processes. The purpose in most cases is to determine the features of the interaction region i.e. of the region where hadrons are produced. Let us suppose that function $\tilde{\rho}(\mathbf{x})$ is the density of some kind of hadrons, say of negative pions, just after they have been produced. Knowing

\footnotetext{
* Supported in part by the KBN grant 2P03B 09322
} 
this function one would know the geometry of the interaction region. According to textbook formulae $\tilde{\rho}(\mathbf{x})$ is proportional to the diagonal elements of the density matrix ${ }^{1}$ in the coordinate representation $\rho_{c}(\mathbf{x} ; \mathbf{x})$. In multiple particle production processes, however, these matrix elements cannot be directly measured - there is no ruler to measure the interaction region. One measures momenta and uses the relation between the density matrices in the coordinate and the momentum representations

$$
\rho_{c}(\mathbf{x} ; \mathbf{x})=\int \frac{d^{3} p_{1} d^{3} p_{2}}{(2 \pi)^{3}} \rho\left(\mathbf{p}_{1} ; \mathbf{p}_{2}\right) e^{i \mathbf{q} \mathbf{x}},
$$

where

$$
\mathbf{q}=\mathbf{p}_{1}-\mathbf{p}_{2}
$$

Thus, in order to determine the shape of the interaction region it is necessary to know both the diagonal and the out-of-diagonal elements of the density matrix in the momentum representation. The diagonal elements are easily obtained, because they are proportional to the density of the single particle momentum distribution $\Omega(\mathbf{p})$. The only thing one can do to get information about the out-of-diagonal elements is to measure the two-body, three-body etc. momentum distributions. In general it is not possible to express these distributions in terms of the single particle density matrix. Making, however, the usual assumption that the $k$-particle density matrix can be approximated by the symmetrized product of single particle density matrices (see, e.g., [1, 2, 3]), one can determine from the momentum distributions of up to $k$ particles the functions

$$
\Re\left[\rho\left(\mathbf{p}_{1} ; \mathbf{p}_{2}\right) \rho\left(\mathbf{p}_{2} ; \mathbf{p}_{3}\right) \ldots \rho\left(\mathbf{p}_{k} ; \mathbf{p}_{1}\right)\right],
$$

where $\Re$ stand for real part of. Thus from single particle momentum distributions one gets the diagonal elements $\rho\left(\mathbf{p}_{1} ; \mathbf{p}_{1}\right)$ as already mentioned. Adding the information about two-body momentum distribution one finds further $\rho\left(\mathbf{p}_{1} ; \mathbf{p}_{2}\right) \rho\left(\mathbf{p}_{2} ; \mathbf{p}_{1}\right)=\left|\rho\left(\mathbf{p}_{1} ; \mathbf{p}_{2}\right)\right|^{2}$ - the absolute values of the out-of-diagonal matrix elements, but not their phases. Measuring more-particle momentum distributions one finds some further information abut the phases, but not all of it. It turns out that it is possible to include in the single particle density

\footnotetext{
${ }^{1}$ It is simplest to use the interaction picture. Then the density matrix after freeze-out is time-independent
} 
matrix elements an additional phase factor without changing the predicted momentum distributions. The choice of this factor, which is unconstrained by the data, has a dramatic effect on the size and shape of the interaction region which one deduces from the momentum measurements. It is the purpose of the present paper to investigate this effect in a systematic way.

In the next section the phase ambiguity is explicitly formulated. Its effect on the size and shape of the interaction region is discussed in Section 3. Two specific examples are presented in Sections 4 and 5. Our conclusions are summarized in the last section.

\section{Group of transformations leaving the mo- mentum distributions invariant}

It is easily checked that making the replacement

$$
\rho\left(\mathbf{p}_{1} ; \mathbf{p}_{2}\right) \rightarrow \rho_{\phi}\left(\mathbf{p}_{1} ; \mathbf{p}_{2}\right)=e^{i\left[\phi\left(\mathbf{p}_{1}\right)-\phi\left(\mathbf{p}_{2}\right)\right]} \rho\left(\mathbf{p}_{1} ; \mathbf{p}_{2}\right),
$$

where $\phi(\mathbf{p})$ is an arbitrary, real-valued ${ }^{2}$, function, one leaves all the observables (3) unchanged. Thus the measured momentum distributions are invariant with respect to the transformation given by (41). In other words, the measured momentum distributions yield no argument for or against any choice of $\phi(\mathbf{p})$.

Before analyzing the effect of this phase ambiguity on the determination of the interaction region let us recall the standard procedure.

In order to obtain information on the interaction region from the BoseEinstein correlations in multiple particle production one usually applies the formula (cf. e.g. the review [4])

$$
\rho\left(\mathbf{p}_{1} ; \mathbf{p}_{2}\right)=\int d^{4} X S(K, X) e^{i q X}
$$

where

$$
K=\frac{1}{2}\left(p_{1}+p_{2}\right), \quad X=\frac{1}{2}\left(x_{1}+x_{2}\right)
$$

\footnotetext{
${ }^{2}$ When $\phi$ is real-valued, matrix $\rho_{\phi}$ is hermitian and has trace one, thus it can be interpreted as a single particle density matrix.
} 
and $S(K, X)$ is known as the emission function. It follows from the hermiticity of the density matrix that the emission function is real-valued. It is constructed by analogy with the Wigner function and is interpreted as the position and momentum distribution of the produced hadrons [4], [5]. As is well known, this interpretation cannot be exact ${ }^{3}$. We will accept it here, however, without further discussion.

Another well-known difficulty (see e.g. 8]) in the studies of the interaction region is that the Fourier transform (5) cannot be inverted. The reason is that the density matrix on the left-hand side is known only for the on-massshell values of the four-momenta $p_{1}, p_{2}$. In general, there is an infinite variety of different emission functions which correspond to a given density matrix. In order to avoid getting involved with this difficulty we limit our discussion to models, where the emission function can be unambiguously expressed by the Wigner function. Two such cases have been much discussed. When all the identical particles are produced simultaneously at some time $t=0$, one can choose

$$
S(K, X)=\delta\left(X_{0}\right) W(K, \mathbf{X}),
$$

where $W$ is the Wigner function corresponding to the time-independent (interaction representation) density matrix in the momentum representation. When the distribution of longitudinal momenta is weakly correlated with the distribution of transverse momenta, one can consider the transverse emission function $S_{T}\left(\mathbf{K}_{T}, \mathbf{X}_{T}\right)$ as the Wigner function corresponding to the transverse factor $\rho_{T}\left(\mathbf{p}_{T 1} ; \mathbf{p}_{T 2}\right)$ in the density matrix ${ }^{4}$. We will thus use the relation

$$
W_{\phi}(\mathbf{K}, \mathbf{X})=\int \tilde{d} \mathbf{q} \rho_{\phi}(\mathbf{K}, \mathbf{q}) e^{i \mathbf{q} \mathbf{X}},
$$

where $\rho_{\phi}(\mathbf{K}, \mathbf{q}) \equiv \rho_{\phi}\left(\mathbf{p}_{1} ; \mathbf{p}_{2}\right), \tilde{d} \mathbf{q} \equiv d \mathbf{q} /(2 \pi)^{l}$ and $d \mathbf{q}=d^{l} q$ with $l$ being the number of dimensions considered ( the vectors are two- or three-dimensional depending on the model). The Wigner function $W_{\phi}(\mathbf{K}, \mathbf{X})$, given by (8), will be used to define the interaction region.

\footnotetext{
${ }^{3}$ Taken literally, it contradicts the uncertainty principle. The solution is also well known: one has to perform an adequate "smearing" of $S(K, X)$ bringing it into agreement with the requirements of quantum mechanics.

${ }^{4}$ For an example see Section 5.
} 


\section{The phase and the moments of the $X$ dis- tribution}

In order to study the effects of the full group of transformations (44), let us first discuss the moments of the distribution of $\mathbf{X}$. The $K$-dependent averages, for a given choice of the phase $\phi$, are denoted by

$$
\langle g(\mathbf{X})\rangle_{\phi}(\mathbf{K})=\frac{\int d \mathbf{X} W_{\phi}(\mathbf{K}, \mathbf{X}) g(\mathbf{X})}{\int d \mathbf{X} W_{\phi}(\mathbf{K}, \mathbf{X})}
$$

and the full averages by

$$
\langle\langle g\rangle\rangle_{\phi}=\int d \mathbf{K} d \mathbf{X} \mathbf{W}_{\phi}(\mathbf{K}, \mathbf{X}) g(\mathbf{X}) .
$$

Since in the latter average the Wigner function has been integrated over $\mathbf{K}$, there is no problem with the uncertainty principle ${ }^{5}$. It is convenient to introduce the notation

$$
\begin{aligned}
\langle\mathbf{X}\rangle_{\phi=0}(\mathbf{K}) & =\mathbf{r}_{0}(\mathbf{K}) ; & \left\langle\mathbf{X}^{2}\right\rangle_{\phi=0} & =R_{0}^{2}(\mathbf{K}) \\
\langle\langle\mathbf{X}\rangle\rangle_{\phi=0} & =\mathbf{r}_{0} ; & \left\langle\left\langle\mathbf{X}^{2}\right\rangle\right\rangle_{\phi=0} & =R_{0}^{2} .
\end{aligned}
$$

Using the definition (8) and the identity (one dimension, $n=0,1, \ldots$ )

$$
\int d x x^{n} e^{i q x}=(-i)^{n} \frac{\partial^{n}}{\partial q^{n}} \int d x e^{i q x}=2 \pi(-i)^{n} \frac{\partial^{n}}{\partial q^{n}} \delta(q) .
$$

one finds that the denominator on the right-hand side of (9) equals $\rho_{\phi}(\mathbf{K}, 0)$, and

$$
\left\langle X_{j}^{n}\right\rangle_{\phi}(\mathbf{K})=\frac{i^{n}}{\rho_{\phi}(\mathbf{K}, \mathbf{0})}\left(\frac{\partial^{n}}{\partial q_{j}^{n}} \rho_{\phi}(\mathbf{K}, \mathbf{q})\right)_{\mathbf{q}=0},
$$

which is easily generalized to

$$
\left\langle\prod_{j} X_{j}^{n_{j}}\right\rangle_{\phi}(\mathbf{K})=\frac{i^{n}}{\rho_{\phi}(\mathbf{K}, \mathbf{0})}\left(\frac{\partial^{n}}{\prod_{j} \partial q_{j}^{n_{j}}} \rho_{\phi}(\mathbf{K}, \mathbf{q})\right)_{\mathbf{q}=0},
$$

where $n=\sum_{j} n_{j}$.

\footnotetext{
${ }^{5}$ There may be, however, problems with the interpretation of the averages $\langle g(\mathbf{X})\rangle(\mathbf{K})$.
} 
When evaluating these averages we will need derivatives of the phase factor

$$
\phi\left(\mathbf{p}_{1}\right)-\phi\left(\mathbf{p}_{2}\right)=\phi\left(\mathbf{K}+\frac{\mathbf{q}}{2}\right)-\phi\left(\mathbf{K}-\frac{\mathbf{q}}{2}\right)=2 \sinh \left(\frac{\mathbf{q} \cdot \nabla}{2}\right) \phi(\mathbf{K}),
$$

These formulae allow to calculate all moments $\left\langle\left\langle X_{j}^{n}\right\rangle\right\rangle$ in terms of (the integrals of) the derivatives of the single particle density matrix. This gives the full information about the size and shape of the interaction region.

\section{Position-momentum correlations and the size of the interaction region}

We will now discuss in detail two parameters characterizing the interaction region: (i) the average position $\langle\langle\mathbf{X}\rangle\rangle$ and (ii) the averaged square of the size $\left\langle\left\langle\mathbf{X}^{2}\right\rangle\right\rangle-\langle\langle\mathbf{X}\rangle\rangle^{2}$.

Using the formulae ${ }^{6}$

$$
\left[\nabla\left(\phi\left(\mathbf{p}_{1}\right)-\phi\left(\mathbf{p}_{2}\right)\right)\right]_{\mathbf{q}=0}=\nabla \phi(\mathbf{K}) ; \quad\left[\nabla^{2}\left(\phi\left(\mathbf{p}_{1}\right)-\phi\left(\mathbf{p}_{2}\right)\right)\right]_{\mathbf{q}=0}=0
$$

one finds

$$
\begin{aligned}
\langle\mathbf{X}\rangle_{\phi}(\mathbf{K}) & =\mathbf{r}_{0}(\mathbf{K})-\nabla \phi(\mathbf{K}) \\
\left\langle\mathbf{X}^{2}\right\rangle_{\phi}(\mathbf{K}) & =R_{0}^{2}(\mathbf{K})-2 \mathbf{r}_{0}(\mathbf{K}) \cdot \nabla \phi(\mathbf{K})+(\nabla \phi(\mathbf{K}))^{2}
\end{aligned}
$$

and

$$
\begin{aligned}
\langle\langle\mathbf{X}\rangle\rangle_{\phi} & =\mathbf{r}_{0}-\langle\nabla \phi(\mathbf{K})\rangle \\
\left\langle\left\langle\mathbf{X}^{2}\right\rangle\right\rangle_{\phi} & =R_{0}^{2}-2\left\langle\mathbf{r}_{0}(\mathbf{K}) \cdot \nabla \phi(\mathbf{K})\right\rangle+\left\langle(\nabla \phi(\mathbf{K}))^{2}\right\rangle,
\end{aligned}
$$

where the notation

$$
\langle g(\mathbf{K})\rangle=\int d \mathbf{K} \rho_{\phi}(\mathbf{K}, 0) g(\mathbf{K})
$$

\footnotetext{
${ }^{6}$ They are special cases of (16).
} 
has been used.

Function $\langle\mathbf{X}\rangle_{\phi}(\mathbf{K})$ can be interpreted as a measure of the position-momentum correlations. According to formula (4) the phase is fixed, when the function $\phi$ is given. On the other hand, as seen from formula (18), $\phi(\mathbf{p})$ can be also fixed, up to an uninteresting constant, by specifying the position momentum correlation $\langle\mathbf{X}\rangle(\mathbf{K})$.

The variance of $\mathbf{X}$ at fixed $\mathbf{K}$ is of particular interest because it gives the HBT radius, as determined in the usual way from the two-particle distribution in the Gaussian approximation. This can be seen as follows. First, we observe that it is invariant, i.e. does not depend on the choice of $\phi(\mathbf{p})$ :

$$
\left\langle\mathbf{X}^{2}\right\rangle_{\phi}(\mathbf{K})-\left(\langle\mathbf{X}\rangle_{\phi}(\mathbf{K})\right)^{2}=R_{0}^{2}(\mathbf{K})-r_{0}^{2}(\mathbf{K})
$$

It can be therefore calculated independently of the choice of $\phi(\mathbf{p})$. On the other hand, in the Gaussian approximation

$$
\rho(\mathbf{K}, \mathbf{q})=\rho(\mathbf{K}, \mathbf{q}=0) e^{-\mathbf{q}^{2} R_{H B T}^{2}(\mathbf{K}) / 2}
$$

where $R_{H B T}^{2}(\mathbf{K})$ is defined by this relation. Formula (14) implies for this approximation $\langle\mathbf{X}\rangle(\mathbf{K})=0,\left\langle X_{i}^{2}\right\rangle(\mathbf{K})=R_{H B T}^{2}(\mathbf{K})$. According to (23) we thus have

$$
d R_{H B T}^{2}(\mathbf{K})=\left\langle\mathbf{X}^{2}\right\rangle(\mathbf{K})-(\langle\mathbf{X}\rangle(\mathbf{K}))^{2}=R_{0}^{2}(\mathbf{K})-r_{0}^{2}(\mathbf{K}) .
$$

where $d=2,3$ is the number of dimensions.

Note, however, that the variance of $\mathbf{X}$ at fixed $\mathbf{K}$ (and thus also the measured HBT radius) is not the physical size of the interaction region at fixed K. It is only an auxiliary concept. Indeed, according to Heisenberg's uncertainty principle fixing $\mathbf{K}$ implies that the true variance of $\mathbf{X}$ becomes infinite (cf. footnote 3).

The true size of the interaction region, as derived from the Wigner function, is instead determined by the full average, given by

$$
\begin{array}{r}
R_{\text {true }}^{2} \equiv\left\langle\left\langle\mathbf{X}^{2}\right\rangle\right\rangle_{\phi}-\langle\langle\mathbf{X}\rangle\rangle_{\phi}^{2}=R_{0}^{2}-\mathbf{r}_{0}^{2}+ \\
+\left\langle[\nabla \phi(\mathbf{K})]^{2}\right\rangle-\langle(\nabla \phi(\mathbf{K}))\rangle^{2}-2\left(\left\langle\mathbf{r}_{0}(\mathbf{K}) \nabla \phi(\mathbf{K})\right\rangle_{\mathbf{K}}-\mathbf{r}_{0}\langle\nabla \phi(\mathbf{K})\rangle\right)
\end{array}
$$

One sees that this quantity explicitly depends on $\phi(\mathbf{p})$ and so does the interpretation of the HBT measurements. 
The three pairs of terms on the right-hand side are easily identified as: the variance at fixed $\phi=0$, the variance of $\nabla \phi$, and twice the unnormalized correlation coefficient for $r_{0}(\mathbf{K})$ and $\nabla \phi$. The variance of $\phi$ increases the observed variance. The correlation may have either sign. In particular, it may be negative and so large in absolute value that it reduces the observed variance below the variance at $\phi=0$. This is not surprising. Suppose that the variance for some density matrix $\rho_{0}$ is smaller than for $\rho_{\phi}$. Then one could start with $\rho_{\phi}$ and go over to the original $\rho_{0}$ by introducing a suitable phase. This change of phase reduces the variance.

Equating to zero the (variational) derivative of the right-hand side of equation (26) with respect to $\nabla \phi$ one finds the condition for the variance of $\mathbf{X}$ (i.e. the size of the interaction region) to be minimal. It turns out that the minimum is achieved when $\phi$ is chosen such that the average of $\mathbf{X}$ at fixed $\mathbf{K}$ does not depend on $\mathbf{K}$ :

$$
\langle\mathbf{X}\rangle_{\phi}(\mathbf{K})=\mathbf{r}_{0}(\mathbf{K})-\nabla \phi(\mathbf{K})=\mathbf{a}
$$

where $\mathbf{a}$ is a constant vector. In other words, the minimal size is obtained when the phase is chosen such that there is no position-momentum correlation. It is clear from (27) that one can always find $\phi$ which satisfies this condition.

Substituting (27) into (18) one finds that the minimal size of the system (for the given momentum distribution) is

$$
\left[\left\langle\left\langle\mathbf{X}^{2}\right\rangle\right\rangle_{\phi}-\langle\langle\mathbf{X}\rangle\rangle_{\phi}^{2}\right]_{\min }=R_{0}^{2}-\left\langle\mathbf{r}_{0}^{2}(\mathbf{K})\right\rangle=<R_{H B T}^{2}(\mathbf{K})>
$$

For a generic $\phi(\mathbf{p})$ the interaction regions at various $\mathbf{K}$ 's are shifted with respect to each other. This increases the overall, integrated over $\mathbf{K}$, interaction region. Condition $\langle\mathbf{X}\rangle_{\phi}(\mathbf{K})=\mathbf{a}$ puts all these partial integration regions, as well as possible, on top of each other. This minimizes the overall, calculated interaction region. The minimum is just the average over $\mathbf{K}$ of expression (23). We thus conclude that the HBT radius, averaged over $\mathbf{K}$, corresponds to the minimal possible size of the interaction region.

In order to find the moments up to order $2 m$ it is enough to know the odd-order derivative of $\phi$ up to $\nabla^{2 m-1} \phi$. Thus $\langle\mathbf{X}\rangle$ and $\left\langle X_{i} X_{j}\right\rangle$ can be calculated when $\nabla \phi$ is known. Knowing moreover $\nabla^{3} \phi$ one can calculate all the moments up to fourth order. In general it is not possible to calculate the Wigner function $W_{\phi}$ in closed form. We will discuss, however, two instructive special cases. 


\section{Example I}

We shall consider only the transverse variables. Choosing

$$
\phi(\mathbf{p})=a+\sum_{i} b_{i} p_{i}+\sum_{i} c_{i} p_{i}^{2}
$$

with $a, b_{i}, c_{i}$ constant $(i=x, y)$, one obtains

$$
\phi\left(\mathbf{p}_{1}\right)-\phi\left(\mathbf{p}_{2}\right)=\sum_{i} q_{i}\left(b_{i}+2 c_{i} K_{i}\right) \equiv \sum_{i} q_{i} V_{i}
$$

According to formula (18) this is the most general case where the phase $\phi\left(\mathbf{p}_{1}\right)-\phi\left(\mathbf{p}_{2}\right)$ is linear in $q$. In this case, from (5), we can write

$$
\rho_{\phi}\left(\mathbf{p}_{1} ; \mathbf{p}_{2}\right)=\int d^{2} X S(\mathbf{K}, \mathbf{X}) e^{i \mathbf{q}(\mathbf{X}+\mathbf{V})}
$$

Changing the integration variables $\mathbf{X}$ into $\mathbf{X}+\mathbf{V}$ one finds the emission function corresponding to the density matrix $\rho_{\phi}$

$$
\left.S_{\phi}(\mathbf{K}, \mathbf{X})=S(\mathbf{K}, \mathbf{X}-\mathbf{V})\right)
$$

Thus, the interaction region is shifted in space-time by $\mathbf{V}$ :

$$
X_{i} \rightarrow X_{i}-b_{i}-2 c_{i} K_{i} .
$$

This formula shows that introducing the phase (29) may have two effects:

- A rigid shift of the interaction region.

- A correlation between the momentum and the space-time position of the emission point of the particle.

The first point is well-known and not really disturbing. It has been discussed by many authors. The second has some relation to the notion of the homogeneity region [6, 7], but to the best of our knowledge its relation to the phase of the density matrix has not been pointed out.

In order to show that these considerations are not purely academic we will now consider a specific model [9, 10, 11] where the emission function is 
derived from a physical picture. The main assumptions, at the classical level, are

$$
\begin{gathered}
K^{\mu}=\lambda X^{\mu} \quad \text { for } \quad \mu=0,1,2,3, \\
X_{0}^{2}-X_{\|}^{2}=\tau^{2},
\end{gathered}
$$

where $\tau$ is a constant. Assumptions (34) and (35) for the temporal and longitudinal components were introduced by Bjorken [12] and Gottfried [13] in their boost invariant description of the multiple particle production processes. The full assumption (34) was introduced by Csörgö and Zimányi [14] who used it to explain why in $e^{+} e^{-}$annihilations the correlation function depends on $q^{2}$ only. The motivation in [9] was to explain why the estimated radii of the interaction region decrease rapidly with increasing particle mass. Let us add the definition of the transverse mass

$$
M_{T}^{2}=K_{0}^{2}-K_{\|}^{2}
$$

Note that, while for a particle of given mass $m_{T}^{2}=m^{2}+p_{T}^{2}$ depends on the transverse momenta, here $K^{2}$ is not fixed and thus $M_{T}^{2}$ depends on the longitudinal and temporal components of momentum. Assumptions (34) and (35) imply

$$
\lambda=\frac{M_{T}}{\tau}
$$

It is easy to see qualitatively, why in this model the measured radius of the interaction region is a decreasing function of the particle mass. Let us make the crude assumption that the momentum distributions are the same for all the particles. Then the distribution of $M_{T} X$ is universal and since $M_{T}$ is bigger for heavy particles, the corresponding distribution in $X$ is narrower.

Let us consider the transverse part of the emission function proposed in [10]:

$$
S_{T}=\exp \left[-\frac{\mathbf{X}_{T}^{2}}{2 r_{T}^{2}}-\frac{\left(\mathbf{K}_{T}-\frac{M_{T}}{\tau} \mathbf{X}_{T}\right)^{2}}{2 \delta_{T}^{2}}\right] .
$$

It can be interpreted as a Wigner function ${ }^{7}$ and thus our previous discussion

\footnotetext{
${ }^{7}$ At fixed $M_{T}$
} 
is applicable to it. This Wigner function can be rewritten in the form

$$
S_{T}=\exp \left[-\frac{\phi_{T}^{2}}{2 R_{D}^{2}}-\frac{\left(X_{T}-\phi_{T}\right)^{2}}{2 R_{\phi}^{2}}\right],
$$

where

$$
\begin{aligned}
R_{\phi} & =\frac{r_{T}}{\sqrt{1+\mu^{2}}} \\
\phi_{T} & =r_{T} \frac{\mu}{1+\mu^{2}} \frac{\mathbf{K}_{T}}{\delta_{T}} \\
R_{D} & =\mu R_{\phi}
\end{aligned}
$$

with

$$
\mu=\frac{r_{T} M_{T}}{\tau \delta_{T}} .
$$

$S_{T}$ and $\phi_{T}$ are of the form discussed in the preceding section ${ }^{8}$. We thus conclude that the measured HBT radius is $R_{\phi}$, whereas the true size of the interaction region, according to (26) , is

$$
R_{\text {true }}^{2}=R_{\phi}^{2}+R_{D}^{2}=r_{T}^{2} .
$$

Thus, while $R_{\phi}$ decreases with increasing transverse mass $M_{T}, R_{\text {true }}$ does not depend on the transverse mass. This explains why the measured HBT radii may be very small at large particle (transverse) mass, while the actual emission region is mass independent.

\section{Example II}

As an instructive, more complicated, example of the phase we now consider

$$
\phi(\mathbf{p})=\frac{4}{3} \sum_{i} a_{i}^{-3} p_{i}^{3} .
$$

\footnotetext{
${ }^{8}$ Because of the presence of $M_{T}$ this is an approximation, valid when only two-body symmetrization is used and/or when the variance of $M_{T}$ can be neglected.
} 
where $i=x, y$ (we consider only transverse dimensions). In order to keep the discussion simple we will start with the Gaussian density matrix

$$
\rho\left(\mathbf{p}_{1}, \mathbf{p}_{2}\right)=\frac{1}{2 \pi \Delta^{2}} e^{-\frac{\mathbf{K}^{2}}{2 \Delta^{2}}} e^{-\frac{1}{2} R^{2} \mathbf{q}^{2}}
$$

With this choice we obtain

$$
\rho_{\phi}\left(\mathbf{p}_{1}, \mathbf{p}_{2}\right)=\rho\left(\mathbf{p}_{1}, \mathbf{p}_{2}\right) e^{i\left[q_{x}\left(4 K_{x}^{2}+q_{x}^{2} / 3\right) / a_{x}^{3}+q_{y}\left(4 K_{y}^{2}+q_{y}^{2} / 3\right) / a_{y}^{3}\right]}
$$

where we have used the identity $p_{1}^{2}+p_{1} p_{2}+p_{2}^{2}=3 K^{2}+\frac{1}{4} q^{2}$ valid for both the $x$ and the $y$ components. Using the formulae of the previous section we find from (46) and (47) the average shift of the $\mathbf{X}$ distribution:

$$
<X_{j}>\left(K_{j}\right)=-4 K_{j}^{2} / a_{j}^{3}
$$

where $j=x, y$.

From relation (47) we obtain

$$
S_{\phi}(\mathbf{K}, \mathbf{X})=\int \frac{d^{2} \mathbf{q}}{4 \pi^{2}} e^{i \mathbf{q} \mathbf{X}} \rho_{\phi}\left(\mathbf{p}_{1}, \mathbf{p}_{2}\right)=s_{x \phi}\left(K_{x}, x\right) s_{y \phi}\left(K_{y}, y\right)
$$

with

$s_{j \phi}\left(K_{j}, X_{j}\right)=\frac{e^{-\frac{K_{j}^{2}}{2 \Delta^{2}}}}{\Delta \sqrt{2 \pi}} \int_{-\infty}^{+\infty} \frac{d q_{j}}{2 \pi} \exp \left[-\frac{1}{2} R^{2} q_{j}^{2}+i q_{j} X_{j}+i \frac{1}{3 a_{j}^{3}}\left(12 K_{j}^{2} q_{j}+q_{j}^{3}\right)\right]$.

The integral can be evaluated in terms of the Airy function. To simplify notation, from now on we drop the subscript $j$.

Introducing a new variable $z$ by

$$
q=a\left(z-\frac{i}{2} \omega^{2}\right)
$$

where

$$
\omega=a R
$$

we obtain

$$
s_{\phi}(K, X)=\frac{a e^{-\frac{K^{2}}{2 \Delta^{2}}}}{\Delta \sqrt{2 \pi}} \int_{-\infty}^{+\infty} \exp \left[\frac{i}{3} z^{3}+i A z+B\right] \frac{d z}{2 \pi}
$$

where 


$$
\begin{aligned}
A & =a[X-<X>(K)]+\omega^{4} / 4 \equiv a \hat{X}+\omega^{4} / 4 \\
B & =\frac{\omega^{2}}{2}\left[a \hat{X}+\omega^{4} / 6\right]
\end{aligned}
$$

The integral can be expressed in terms of the Airy function (cf. [15] for an equivalent formula)

$$
A i(u)=\frac{1}{2 \pi} \int_{-\infty}^{+\infty} e^{\frac{i}{3} t^{3}+i u t} d t
$$

The result is

$$
s_{\phi}(K, X)=\frac{a}{\Delta \sqrt{2 \pi}} e^{\frac{-K^{2}}{2 \Delta^{2}}} e^{B} A i(A)
$$

One sees from this formula that, for fixed $K$, the variation of $\hat{X}$, i.e. spread of $X$ around the average $\langle X\rangle(K)$ is determined by two parameters: $a$ and $\omega$. From the known asymptotic expansion of $A i(z)$ one can deduce that in the limit $a \rightarrow \infty$ one recovers the original Gaussian distribution. In the limit $a \rightarrow 0$ the distribution becomes singular. Formally one obtains

$$
s_{\phi}(K, X) \rightarrow \frac{a e^{\frac{-K^{2}}{2 \Delta^{2}}}}{\Delta \sqrt{2 \pi}} A i(a \hat{X}) .
$$

and the averages of $\hat{X}$ and $\hat{X}^{2}$ do not exist.

The Airy function is negative for some values of $A$, showing explicitly that the function $s_{\phi}(K, X)$ cannot be literally interpreted as a distribution function. This is illustrated in Fig. 1, where $s_{\phi}(K, X)$ is plotted versus $a \hat{X}$ for $K=0$ and $\omega=0,1,2$. For $\omega=0$ it is an Airy function. With increasing $\omega$ the maximum moves towards $\hat{X}=0$, the oscillations get dumped and already at $\omega=2$ the curve is almost a Gaussian.

The formulae from Section 3 yield

$$
\left\langle x_{j}\right\rangle=-\frac{4 \Delta^{2}}{a_{j}^{3}} ; \quad \sigma^{2}\left(x_{j}\right)=R^{2}+2\left\langle x_{j}\right\rangle^{2}
$$

The net result is a shift of the interaction region and an increase of its size. 


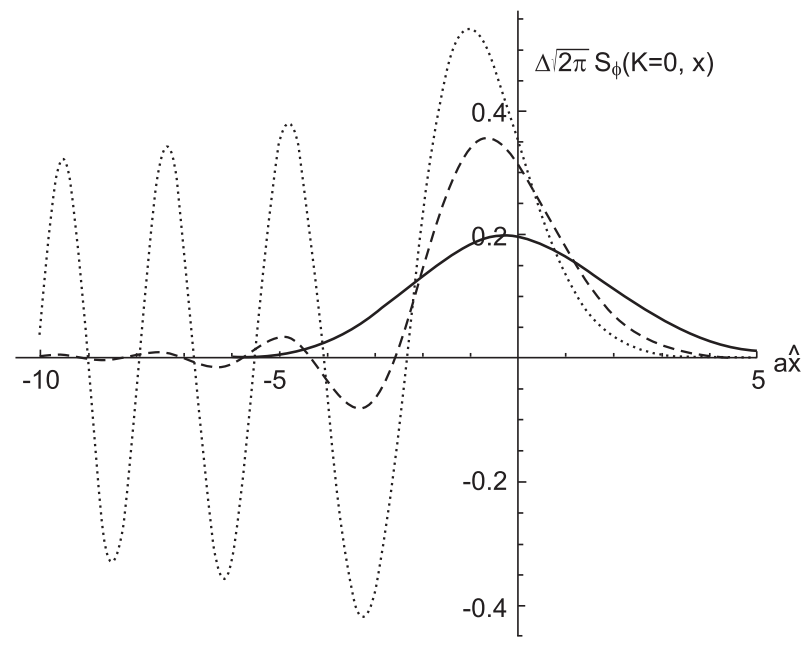

Figure 1: Emission function $s_{\phi}(K, X)$ versus $a \hat{X}$. Dotted line: $\omega=0$, dashed line: $\omega=1$, full line: $\omega=2$.

\section{Conclusions}

For models where particle production is uncorrelated except for the BoseEinstein correlations, we have identified a group of transformations of the phase of the single particle density matrix which leave all the the single- and multiparticle momentum distributions invariant. The effect of the resulting uncertainty of the size and shape of the interaction region as deduced from the measured momentum distributions is systematically discussed. Explicit formulae taking into account this uncertainty are derived for the moments of the $\mathbf{X}$ distribution. It is shown that the phase ambiguity can have a dramatic effect on the parameters characterizing the interaction region. Two explicit examples are discussed in detail.

\section{References}

[1] J. Karczmarczuk, Nucl.Phys. B78(1974)370.

[2] A.Bialas and A.Krzywicki, Phys.Letters B354(1995)134.

[3] T. Csörgö, Heavy Ion Physics, 15(2002)1.

[4] U.A. Wiedemann and U. Heinz, Phys. Rep. 319(1999)145. 
[5] S. Pratt, Phys. Rev. Letters, 53(1984)1219.

[6] M.G. Bowler, Z. Phys. C29(1985)617.

[7] Yu.M. Sinyukov, in J. Letessier, H.H.Gutbrod and J. Rafelski eds., Hot Hadronic Matter Theory and Experiment, Plenum press New York (1995) p. 309.

[8] K.Zalewski, Acta. Phys. Pol. B34(2003)3379.

[9] A.Bialas and K.Zalewski, Acta Phys. Pol. B30(1999)359.

[10] A.Bialas, M.Kucharczyk, H.Palka and K.Zalewski, Phys. Rev. D62(2000)114007.

[11] A.Bialas, M.Kucharczyk, H.Palka and K.Zalewski, Acta Phys. Pol. B32(2001)2901.

[12] J.D. Bjorken, Phys. Rev. D7(1973)282.

[13] K. Gottfried, Phys. Rev. Letters 32(1974)975.

[14] T. Csörgö and J. Zimányi, Nucl. Phys. A517(1990)588.

[15] M. Abramovitz and I.A. Stegun eds. Handbook of Mathematical Functions Dover, New York p. 447. 